\title{
Numerical Modelling and Experimental Investigation of a Single and a Compound Pendulum Impact Dynamic Vibration Absorbers
}

\author{
M.F. Younes*
}

\begin{abstract}
This paper deals with vibration reduction of a mechanical vibrating system using passive impact vibration absorber (IVA). The damping potentials of a single mass IVA (SIVA) and a compound pendulum IVA (PIVA) are studied. The numerical and the experimental investigations are carried in free vibration modes. The influence of the IVA's configuration parameters such as the mass ratio, restitution coefficient, clearance, and the pendulum length on the vibration reduction for the main system were studied. A single degree of freedom (SDOF) oscillator equipped with a PIVA is designed and fabricated to conduct the experimental tests. The results show that the efficiency of the IVA depends mostly on the number of effective collisions rather than the aggregate number of impacts. The IVA's clearance and the mass ratio are important design parameters for the SIVA. In addition, to the pendulum length for the PIVA. A good combination of these design parameters accomplishes the higher vibration suppression capacity. The results demonstrate that it is recommended to take the IVA's mass ratio lying around 0.1. In addition, low or high values of the restitution coefficient do not improve the damping capacity. Moreover, it is recommended to select the relative clearance between 0.4 and 0.6 for the SIVA and between 1.3 to 1.5 with frequency ratio around 0.4 for the PIVA to maximize damping. The PIVA with suitable parameters can attenuate vibrations much better than SIVA by about $50 \%$.
\end{abstract}

Keywords: impact damper, dynamic absorber, passive control, coefficient of restitution, vibration attenuation.

\section{Introduction}

It is important to reduce the unwanted vibrations that cause fatigue and/or failure of mechanical systems such as turbine blades, machine tools, fluid pipes, and television towers. Active and passive vibration control systems are recommended techniques for reducing undesired vibration in many applications. Active dampers are not proper for all applications due to cost, power requirements, environment, etc. Therefore, passive damping techniques such as viscous dampers, viscoelastic and friction dampers, and dynamic absorbers are applicable choices.

Viscous and viscoelastic dampers are not reliable due to temperature and due to wear limitations. The dynamic absorber is a good choice especially for applications with very high or very low temperatures. An impact vibration absorber (IVA) is more efficiently than the conventional dynamic absorbers [1]. It consists of an impact mass, which is mounted on the main vibrating system and periodically collides with it. The transfer of momentum to the impact mass from the main system and the reduction of energy in each impact decreases vibrations of the main system [2-4]. The simple design and damping advantages for this type

Department of Mechanical Design, Faculty of Engineering (Elmataria), Helwan University, Cairo, Egypt, mf.fahmy@gmail.com 
of vibration absorbers prompted a significant number of researchers to study its dynamic performance [5-7].

Son et al. [8], introduced active momentum exchange IVA to suppress the first large peak value of the acceleration response due to a shock load. Cheng and Xu [9], studied the effect of the restitution coefficient on the impact damping ratio also they obtained that optimum initial excitation is a monotonically increasing function of damping. Jam and Aref [10], introduced a mathematical model for a PIVA with an elastic fence by using a Hertzian contact model. Ekwaro-Osire et al [11], compared the damping properties of single mass IVA with compound IVA and concentrated on the influence of system parameters on the efficiency of the compound IVA. Vinayaravi et al [12], proposed details of the velocity response for an IVA using the mathematical model which undergoes momentum transfer. Experiments are also carried out on a cantilever beam with IVA and the findings are compared with the theoretical results. Aref and Anooshiravan [13], introduced a simple model of an IVA with a nonlinear spring and a magnetorheological damper. They studied the influences of the current and the impact mass on the vibration reduction of the main structure. Mao et al [14], utilized a discrete element technique to describe and simulate the damping performance of IVA for transient vibrations. Yoshio et al [15], carried out a numerical and experimental study on an IVA with multi vibrators. Semercigil et al [16], studied the effect of the combination of a tuned absorber and IVA on the settling time of the system, the settling time was reduced by about $80 \%$. Li and Darby [17], studied the dynamic performance of IVA and its efficiency and determined a loss factor.

Although the IVA has been applied for a long time, there is still a problem of choosing the parameters that sufficiently stifles the response of the vibratory system in different applications. This paper attempts to report the suitable values of the design parameters of the SIVA and PIVA which maximize the damping capacity. These include the effect of mass ratio, excitation amplitude, clearance size, coefficient of restitution and pendulum length on the vibration reduction. A comparison of the damping characteristics of SIVA and PIVA in free vibration is carried. Finally, experiments of SDOF system equipped with PIVA are carried out to verify the numerical results.

\section{Mathematical Models}

Fig. 1 shows a schematic drawing for the two models, SIVA and PIVA, attached with SDOF main system. While the main system starts to vibrate, the impact mass starts to impact with the sides of the main system. Frequent collisions lead to reduce of the kinetic energy of the system and therefore attenuate the vibration of the main system.

In case of $-d \leq x(t)-x_{1}(t) \leq d$ the mass of SIVA moves with a steady velocity without colliding. Due to small size and lubrication of the clearance, the friction can be ignored. By using Lagrange's equations, the equations of motion of the main system and the SIVA can be expressed as follows:

$$
\begin{aligned}
& \ddot{x}+2 \xi \omega_{n} \dot{x}+\omega_{n}^{2} x=\frac{f(t)}{M} \\
& \mu \ddot{x}_{1}=0
\end{aligned}
$$

where;

$x(t)$ : the displacement of the main system, $x_{1}(t)$ : the displacement of the SIVA's mass, $\mathrm{d}$ : the absorber clearance, $\mathrm{m}$ : the impact mass, $\mathrm{M}$ : the mass of the main system, $\mathrm{c}$ : the damper constant, $\mathrm{k}$ : the spring stiffness, $f(t)$ is the external force, $\mu=\frac{m}{M}$ : the mass ratio, $\omega_{n}=\sqrt{\frac{K}{M}}$ : the natural frequency of the main system and $\zeta=\frac{c}{2 M \omega_{n}}$ : is the damping ratio.

For the PIVA, $\theta(\mathrm{t})$ is the angular displacement of the pendulum mass, in case of $|L \sin \theta| \leq d$ the pendulum mass moves without any impact. Using Lagrange's equations, the equations of motion of the main system and PIVA may be expressed as: 


$$
(M+m) \ddot{x}+m L\left(\ddot{\theta} \cos \theta-\dot{\theta}^{2} \sin \theta\right)+c \dot{x}+k x=f(t)
$$

$$
m L^{2} \ddot{\theta}+m L \ddot{x} \cos \theta+m g L \sin \theta=0
$$

Thus,

$$
\begin{aligned}
& \ddot{x}(1+\mu)+\frac{\mu g}{\omega_{p}^{2}}\left(\ddot{\theta}-\dot{\theta}^{2} \sin \theta\right)+2 \xi \omega_{n} \dot{x}+\omega_{n}^{2} x=\frac{f(t)}{M} \\
& \frac{g}{\omega_{p}^{2}} \ddot{\theta}+\ddot{x} \cos \theta+g \sin \theta=0
\end{aligned}
$$

where; $\mathrm{L}$ and $\omega_{p}=\sqrt{\frac{g}{L}}$ are the pendulum length and natural frequency.

The condition for the impact between the IVA's mass and the main system can be represented as:

In case of SIVA: $\left|x(t)-x_{1}(t)\right|>d$

In case of PIVA: $\quad|L \sin \theta|>d$

Stereo mechanical model $[18,19]$, is used to simulate the collision of the main system and the impact mass. This model utilizes the coefficient of restitution and the momentum conservation theory to adjust the velocities of the impacting elements. The momentum conservation in the impact process may be written as:

$$
M v^{b}+m v_{1}^{b}=M v^{a}+m v_{1}^{a}
$$

where $v^{a}$ and $v_{1}^{a}$ are the velocity of the main system and the velocity of the impact mass respectively just after impact. $v^{b}$ and $v_{1}^{b}$ are the velocity of the main system and the velocity of the impact mass respectively just before impact. In addition, the restitution coefficient $e$ can be expressed as:

$$
e=-\frac{v^{a}-v_{1}^{a}}{v^{b}-v_{1}^{b}}
$$

By solving Eqs. (9) and (10), the velocities after the impact can be formulated as follows:

$$
\begin{aligned}
& v^{a}=\frac{1}{M+m}\left((M-m e) v^{b}+(1+e) m v_{1}^{b}\right) \\
& v_{1}^{a}=\frac{1}{M+m}\left((m-M e) v_{1}^{b}+(1+e) M v^{b}\right)
\end{aligned}
$$

Thus, in case of SIVA the velocities after the impact can be expressed as follows:

$$
\begin{aligned}
& v^{a}=\frac{1}{1+\mu}\left((1-\mu e) v^{b}+(1+e) \mu v_{1}^{b}\right) \\
& v_{1}^{a}=\frac{1}{1+\mu}\left((\mu-e) v_{1}^{b}+(1+e) v^{b}\right)
\end{aligned}
$$

In case of PIVA, the linear velocity of the pendulum mass can be written as:

$$
v_{1}^{b}=v^{b}+L \dot{\theta}^{b} \text { and } L \dot{\theta}^{a}=v_{1}^{a}-v^{a}
$$

where; $\dot{\theta}^{b}$ and $\dot{\theta}^{a}$ are the pendulum angular velocity just before and after the impact respectively.

Therefore, in case of PIVA the velocities after the impact can be formulated as follows:

$$
\begin{aligned}
v^{a} & =v^{b}+\frac{\mu(1+e)}{1+\mu} L \dot{\theta}^{b} \text { and } v_{1}^{a}=v^{b}+\frac{\mu-e}{1+\mu} L \dot{\theta}^{b} \\
\dot{\theta}^{a} & =\frac{-2 \mu}{1+\mu} e \dot{\theta}^{b}
\end{aligned}
$$

The Runge-Kutta numerical method is utilized to solve simultaneously the equations of motion. These equations are solved with initial excitations from the start to the instant of the collision. The solution stops when an impact event occurs according to Eqs. (7) and (8). The velocities obtained at this time are those just before an impact. Equations (13), (14), (16) and (17) can be used to find the velocities just after the impact, which are the new initial conditions to the next step. The solutions for the equations of motion are continued with the new initial conditions. A new initial condition by the same manner is assigned for each impact. 


\section{Experimental Setup}

As displayed in Fig. 2, the test rig consists of a flexible steel strips acting as a leaf springs and two bases made from steel L-beams. The strips are fixed onto the upper and the lower bases. The lower base was fixed to a stationary base. Two aluminum rectangular plates were mounted on the upper base to act as stoppers to the motion of the pendulum mass. The distance between the stop plates can be modify the absorber's clearance. The pendulum impact mass is connected to a transverse beam using an aluminum rod which is rotating freely. The pendulum mass can be changed by joining or ejecting auxiliary disks in order to modify the impact mass. The tests were carried out by setting the upper base in a certain longitudinal position and then releasing it. The response of the main mass is monitored using an accelerometer mounted on the upper base. The measurements are supplied to a PC by utilizing LabVIEW 5.1 software through the data acquisition card. The tests are performed with and without PIVA. The effect of PIVA clearance and mass ratio on the damping of the main system are studied.

\section{Numerical Results and Discussion}

\subsection{Effects of SIVA on the Vibration Response}

Fig. $\underline{3}$ displays the relative responses of the main system $\left(\frac{x}{x o}\right)$ in time domain corresponding to the relative clearances $\gamma=\frac{d}{x_{o}}=0.1,0.6$ and 0.8 , where $x_{o}$ is the initial displacement given to the main system. The relative responses of the main system decrease compared to the case without SIVA. Furthermore, the relative responses of the main system when $\gamma=0.6$ compared to the relative responses of $\gamma=0.1$ and $\gamma=0.8$ is attenuated in a short time. To get a comprehension of the reasons for this behavior, additional analyses are needed.

Variation of the velocity of the main system $(\mathrm{v})$ and the velocity of the impact mass $\left(\mathrm{v}_{1}\right)$ with time appeared in Figs. 4 and 5 for $\gamma=0.1$ and $\gamma=0.6$ respectively. As shown in Fig. 4 the impact mass moves with the mass of main system in the same direction but with different velocity and numerous collisions happen in that case. Nevertheless, small amount of energy is dissipated, since the relative velocities between the impact mass and the main mass are small. Referring to Fig. 5, it is noted that the relative velocities are increasing because the masses move in an inverse direction, which lead to an increase in the energy lost from the system. The collision that happens while the masses are moving toward each other maximizes the momentum exchange between the IVA and the main system. Figure 6 displays the effect of the relative clearance $\gamma$ on the number of impacts for the SIVA for various values of mass ratio. The largest relative clearance $(\gamma=1)$ has the lowest number of impacts while; the smallest clearance $(\gamma=$ 0.1 ) has the highest number of impacts. On the other hand, the variation of damping ratio with the relative clearance is shown in Fig. 7, where the mass ratios are taken as a parameter. The damping ratio increases initially with clearance, then reaches a peak, and, beyond $\gamma \cong 0.4-$ 0.6 begins to reduce. The reasons behind this is that; at very small relative clearance, collisions happen with low relative velocities which leads to little energy dissipation. As the relative clearance raises the relative velocities raise and these consequences lead to a growing in the damping ratio. On another hand, for larger relative clearance for example $\gamma=1$, little impacts happen and the impact mass goes to a high distance between impacts and therefore, the damping ratio is decreasing. Based on these outcomes, the number of impacts is not a principal key to attenuate the system vibration. Furthermore, the influence of the mass ratio has little effect if the relative clearance is approximately greater than unity. So small mass ratio such as $\mu \cong 0.05$ decreases the momentum exchange between the SIVA and the main system which decreases the effectiveness of the SIVA. Figs. 8 and 9 show the influence of the relative clearance on the number of impacts and damping ratio respectively for various values of the restitution coefficient. As shown in Fig. 8, while the coefficient of restitution increases the number of impacts also increases. Otherwise, referring to Fig. 9 the highest value of the damping ratio is 
attained when e $\cong 0.5$. This can be explained from Figs. 10 and 11 , while fewer impacts take place when $\mathrm{e}=0.5$ as a result of low bounce velocity. There is a high energy loss in each one which increases the total energy dissipation. In addition, for a higher coefficient of restitution, higher number of impacts happen, but the energy loss in each impact is small, this leads to a lower energy dissipation capacity.

\subsection{Effects of PIVA on the Vibration Response}

Fig. 12 displays the influence of the pendulum length on the decay time of the main system. The relative response of the main system for the longest pendulum length $\left(\omega_{p}=0.4 \omega_{n}\right)$ was smaller than that of the shortest one $\left(\omega_{p}=0.8 \omega_{n}\right)$. Furthermore, the PIVA with the largest pendulum length has the lowest settling time and the PIVA with the smallest pendulum length had the larger settling time. Referring to Figs 13 and 14 as the PIVA natural frequency increases from $\omega_{p}=0.4 \omega_{n}$ to $\omega_{p}=0.8 \omega_{n}$, the total number of impacts also increases and there are two impacts in every cycle. Moreover, a smaller PIVA natural frequency boosts the energy dissipation in each collision because the momentum exchange between the masses increases by raising the arm length. The variation of the damping ratio with relative clearance and frequency ratio $\frac{\omega_{p}}{\omega_{n}}$ respectively is illustrated in Figs. 15 and 16 for three values of the initial displacements. From these figures, it can be noticed that the large initial displacement has the highest damping because the higher collisions occur for largest initial displacements. In addition, the damping ratio of the main system reaches its maximum value at relative clearance and frequency ratio around 1.4 and 0.4 respectively. Figure 17 displays the damping ratio for different values of the restitution coefficient. When the restitution coefficient reduces from 0.8 to 0.5 the damping ratio is greatly increasing. While the restitution coefficient reduces from 0.5 to 0.3 , there are a slightly increase in the damping ratio. In addition, decreasing the restitution coefficient leads to increase the required mass ratio to maximize the system damping.

\subsection{Comparison Between a SIVA and a PIVA}

Fig. 18 shows the oscillation of the main system with SIVA and PIVA in time domain with the same value of mass ratio and restitution coefficient. According to the obtained results in the previous sections, the relative clearances were taken as 0.4 and 1.4 which gave a maximum damping for SIVA and PIVA respectively. The displacement amplitude of the main system with PIVA attenuates much faster than that with SIVA. The PIVA with $\omega_{p}=0.4 \omega_{n}$ decreased the settling time by about $50 \%$.

\section{Experimental Results}

The upper base is moved in the longitudinal direction to a certain position and then released. Its response is recorded until complete motion decay is observed. The initial displacement was adjusted through the strain gages stuck on one of the leaf springs connecting the two bases. The damping performance for the cases with and without the PIVA is studied. The influences of the relative clearance and the mass ratio are considered.

Figures 19 and 20 show the relative main system responses in time domain for free vibration modes for two values of the relative clearance $\gamma=1$ and 1.4 respectively. The effect of impact mass on the magnitude of the settling time is illustrated for four values of the mass ratio $\mu=$ $0.02,0.06,0.12$ and 0.16 . From these figures, it can be noticed that the settling time is decreasing and the damping is increasing with mass ratio for any value of the relative clearance. Referring to Fig. 19, when the mass ratio increases to 0.02 , the settling time decreases to $64 \%$ from its value in the case where PIVA is absent. Moreover, the settling time is reduced from $\mathrm{t}=$ $16 \mathrm{sec}$. to $\mathrm{t}=11 \mathrm{sec}$. while the mass ratio is raised from $\mu=0.02$ to $\mu=0.12$; where the time is improved by about $32 \%$. On another hand, for $\mu=0.12$ the settling time is reduced from $\mathrm{t}=11$ sec. to $t=5$ sec. while the relative clearance is raised from $\gamma=1$ to $\gamma=1.4$; the time is decreased 
by about 55\%. Nevertheless, there are a small improvement in the system damping by increasing the mass ratio from 0.12 to 0.16 . Figures 21 and 22 show the relative main system responses in time domain for free vibration modes for two values of mass ratio $\mu=0.12$ and $\mu$ $=0.02$. The effect of PIVA clearance on the magnitude of the settling time is illustrated for four values of the relative clearance $\gamma=0.6,1,1.4$ and 1.6. It can be noticed that the settling time is decreasing and the damping is increasing with the relative clearance. Referring to Fig. 21 for $\gamma$ $=0.6$, the settling time decreases by $52 \%$ from its value where PIVA is absent. Moreover, the settling time is reduced from $\mathrm{t}=13 \mathrm{sec}$. to $\mathrm{t}=5 \mathrm{sec}$. while the relative clearance is raised from 0.6 to 1.4 , it is improved by about $62 \%$. Meanwhile rising the relative clearance does not consistently lead to a decrease in the vibration level which can be seen from Fig. 22. The damping corresponding to $\gamma=1.6 \mathrm{~cm}$ is less than that of $\gamma=1.4$. From these figures, it can be noticed that, the PIVA with the relative clearance of 1.4 and mass ratio of 0.12 has a better performance than that with mass ratio of $=0.02$ for all relative clearance values.

\section{Comparison of PIVA Numerical and Experimental Results}

The results are obtained from numerical and experimental simulations listed in Table 1. Both damping ratios are nearly equal. Results of using numerical simulation are in good agreement with that of the experiments. As a result, it can be concluded that the presented numerical method can precisely describe the impact vibration absorber system responses.

Table.1. Comparison of PIVA numerical and experimental results

\begin{tabular}{|c|c|c|c|}
\hline $\begin{array}{c}\text { Relative } \\
\text { clearance }\end{array}$ & Mass ratio & $\begin{array}{c}\text { Damping ratio } \\
\text { (Experimental) }\end{array}$ & $\begin{array}{c}\text { Damping } \\
\text { ratio } \\
\text { (Numerical) }\end{array}$ \\
\hline \multirow{4}{*}{1} & 0.02 & 0.145 & 0.147 \\
\cline { 2 - 4 } & 0.06 & 0.23 & 0.227 \\
\cline { 2 - 4 } & 0.12 & 0.254 & 0.249 \\
\cline { 2 - 4 } & 0.16 & 0.25 & 0.245 \\
\hline \multirow{4}{*}{1.4} & 0.02 & 0.219 & 0.221 \\
\cline { 2 - 4 } & 0.06 & 0.235 & 0.231 \\
\cline { 2 - 4 } & 0.12 & 0.323 & 0.328 \\
\cline { 2 - 4 } & 0.16 & 0.292 & 0.288 \\
\hline
\end{tabular}

\section{Conclusions}

The theoretical results showed that the effective collision that happens while the masses are moving toward each other maximizes the momentum exchange between the IVA and the main system. Therefore, the number of effective collisions rather than the aggregate number of impacts is the main source that causes the vibration attenuation. For practice use, it is recommended to select the relative clearance between 0.4 to 0.6 for SIVA and between 1.3 to 1.5 for PIVA to maximize the damping capacity.

The damping of the main system increases by reducing the restitution coefficient as a result of high energy dissipation. Furthermore, in practice due to material distortions, very small restitution coefficients are unfavorable. It is recommended to take the restitution coefficient around 0.5 . Increasing the mass ratio leads to a raise in the momentum exchange between the IVA and the main system and therefore increases the vibration attenuation and the efficiency of the IVA. It is recommended to utilize mass ratio around 0.1 . The results showed that, the using of the PIVA leads to more attenuation of the main system vibration compared to the SIVA. In addition, the longest pendulum is widely effective than the shortest one. It is noted that the PIVA with natural frequency ratio around 0.4 was the most effective. The numerical model shows a good agreement with the experimental findings. 


\section{References}

[1] Barbara Blazejczyk-Okolewska, Analysis of an impact damper of vibrations, Chaos, Solitons and Fractals 12, 1983-1988, 2001.

[2] Ekwaro-Osire S. and Desen I. C., Experimental study on an impact vibration absorber, journal of vibration and control, 7, 4, 475-493, 2001.

[3] Svetlana Polukoshko, Vladislav Jevstignejev and Svetlana Sokolova, Impact vibration absorbers of pendulum type, EN OC., 24-29, Rome, Italy, 2011.

[4] Yanchen Du and Shulin Wang, Modeling the fine particle impact damper, international journal of mechanical sciences, 52, 1015-1022, 2010.

[5] Ema S. and Marui E., Damping characteristics of an impact damper and its application, international journal of machine tools and manufacture, 36, 293-306, 1996.

[6] Ehsan E., Yousefi-Koma A. and Ehyaei D., Optimal design of an impact damper for a nonlinear friction-driven oscillator, international journal of mathematical models and methods in applied sciences, 2, 2, 2008.

[7] Xu Z. W., Wang M. Y., and Chen T. N., An experimental study of particle damping for beams and plates, journal of vibration and acoustics, 126, 1, 141-148, 2004.

[8] Son L., Hara S. and Yamada K. , Experiment of shock vibration control using active momentum exchange impact damper, journal of vibration and control, 16, 1, 49-64, 2010.

[9] Cheng J. and $\mathrm{Xu} \mathrm{H.,} \mathrm{Inner} \mathrm{mass} \mathrm{impact} \mathrm{damper} \mathrm{for} \mathrm{attenuating} \mathrm{structure} \mathrm{vibration,}$ international journal of solids and structures, 43, 17, 5355-5369, 2006.

[10] Jam J. E. and Aref A. Fard, Investigating vibratory behavior of nonlinear single unit impact dampers using perturbation method and genetic algorithm, international journal of aerospace sciences, 1, 2, 16-20, 2012, DOI: 10.5923/j.aerospace.20120102.01.

[11] Ekwaro-Osire S., Ozerdim C. and Khandaker M. P. H., Effect of attachment configuration on impact vibration absorbers, experimental mechanics, 46, 669-681, 2006, DOI 10.1007/s11340-006-9516-4.

[12] Vinayaravi R. , Kumaresan D. , Jayaraj K. , Asraff A.K. and Muthukumar R., ,Experimental investigation and theoretical modelling of an impact damper, journal of sound and vibration, 332, 1324-1334, 2013,

\section{[1] htttp://dx.doi.org/ 10.1016/j.jsv.2012.10.032}

[13] Aref Afsharfard and Anooshiravan Farshidianfar, Modeling and analysis of magnetorheological inner mass single unit impact dampers, journal of intelligent material systems and structures, 25, 3, 342-351, 2014

[14] Mao K. M., Wang M. Y., Xu Z. W., and Chen T. N., Simulation and characterization of particle damping in transient vibrations, journal of vibration and acoustics, 126, 2, 202211, 2004.

[15] Yoshio Iwata ,Toshihiko Komatsuzaki ,Satoshi Kitayama and Tatsuya Takasaki, Study on optimal impact damper using collision of vibrators, journal of sound and vibration 361, 66-77, 2016, http://dx.doi.org/10.1016/jisv.2015.09.036

[16] Semercigil SE, Collette F and Huynh D. Experiments with tuned absorber-impact damper combination, journal of sound and vibration, 256, 179-188, 2002.

[17] Li K and Darby AP., An experimental investigation into the use of a buffered impact damper, journal of sound and vibration, 291, 844-860, 2006.

[18] Muthukumar S. and DesRoches R., A Hertz contact model with nonlinear damping for pounding simulation, earthquake engineering and structural dynamics, 35, 811-828, 2006.

[19] Mohamed Gharib and Saud Ghani, Free vibration analysis of linear particle chain impact damper, journal of sound and vibration, 332, 6254-6264, 2013. 

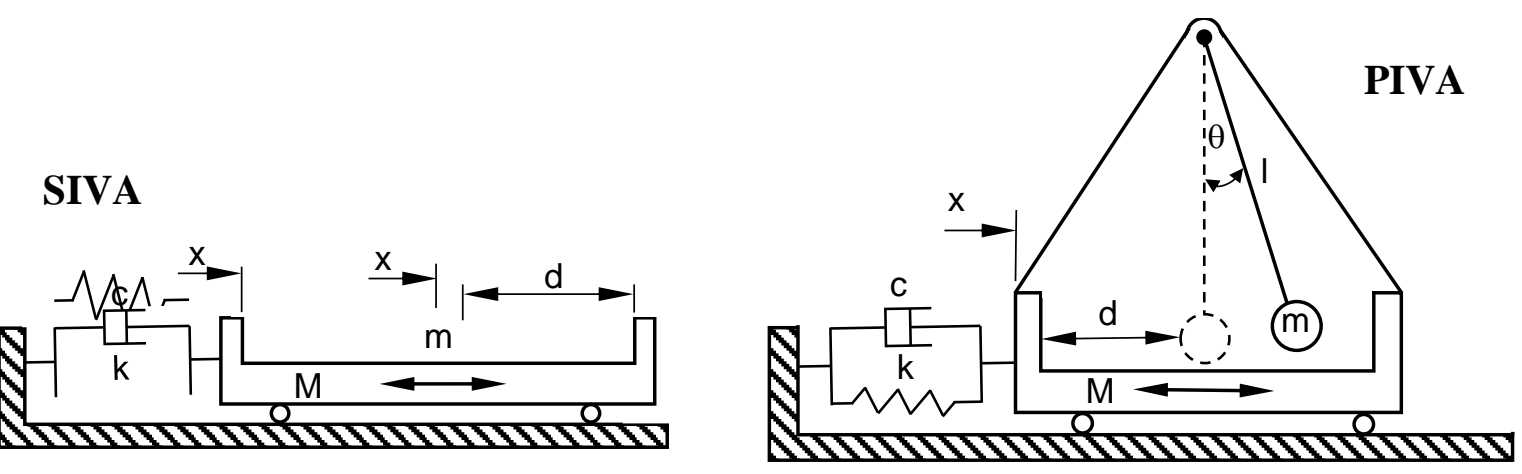

Fig. 1. Schematic diagram of the IVA models

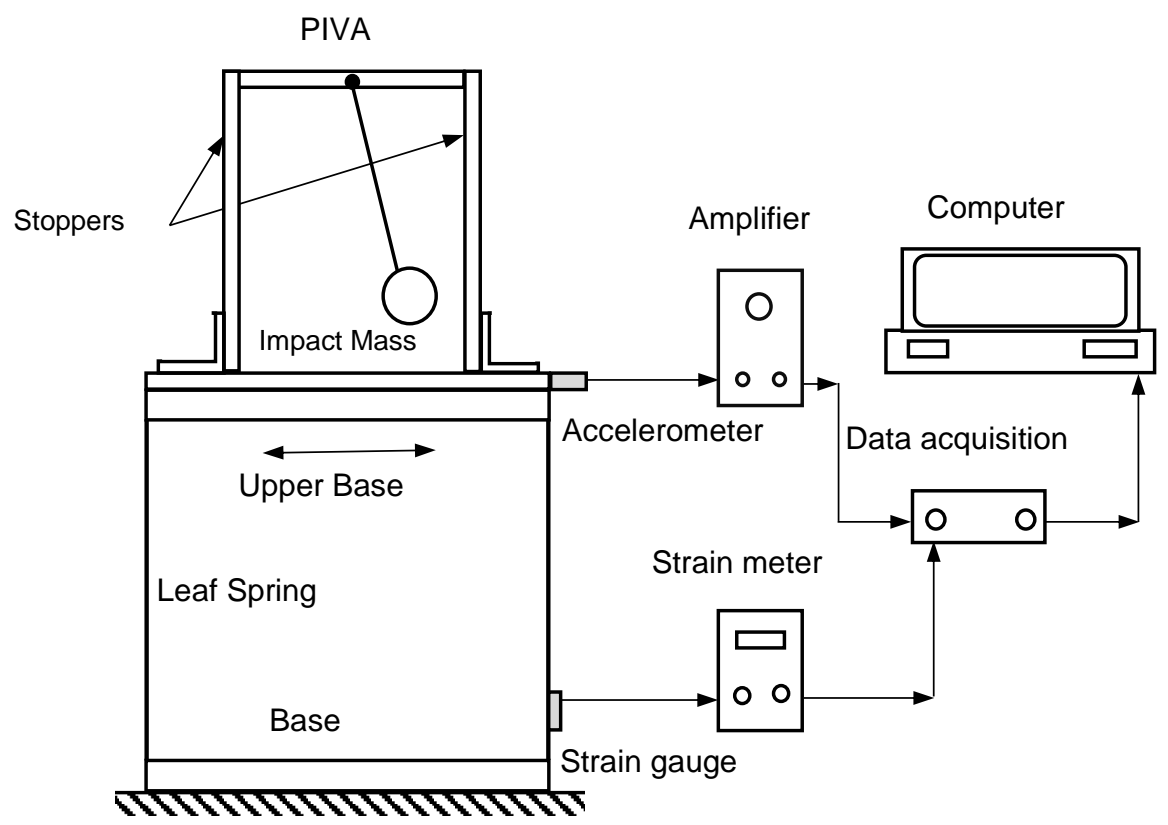

Fig. 2. Schematic of the Experimental Setup

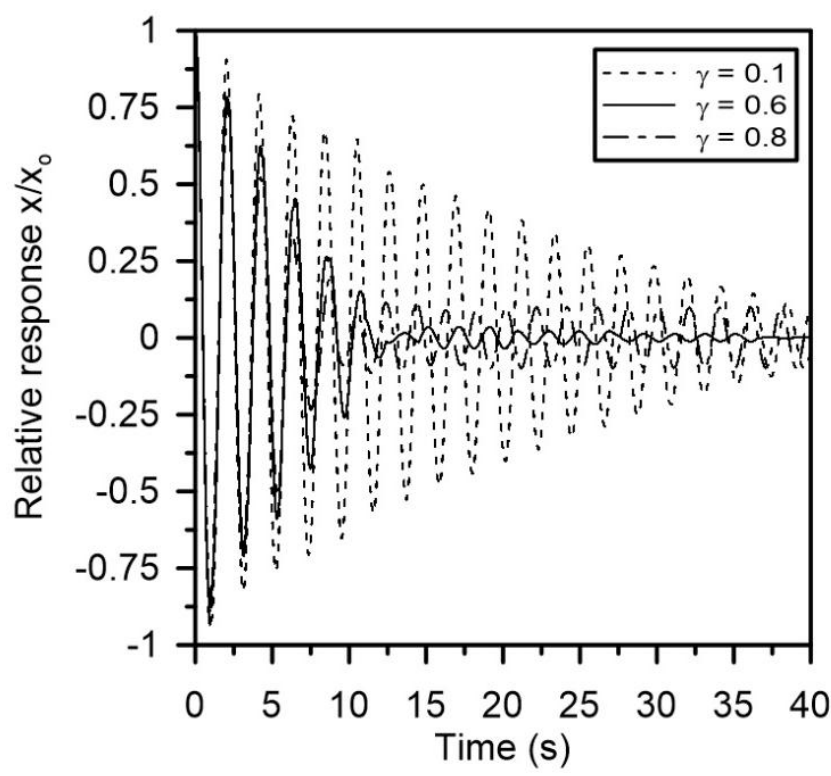

Fig. 3. Relative response for different relative clearances 


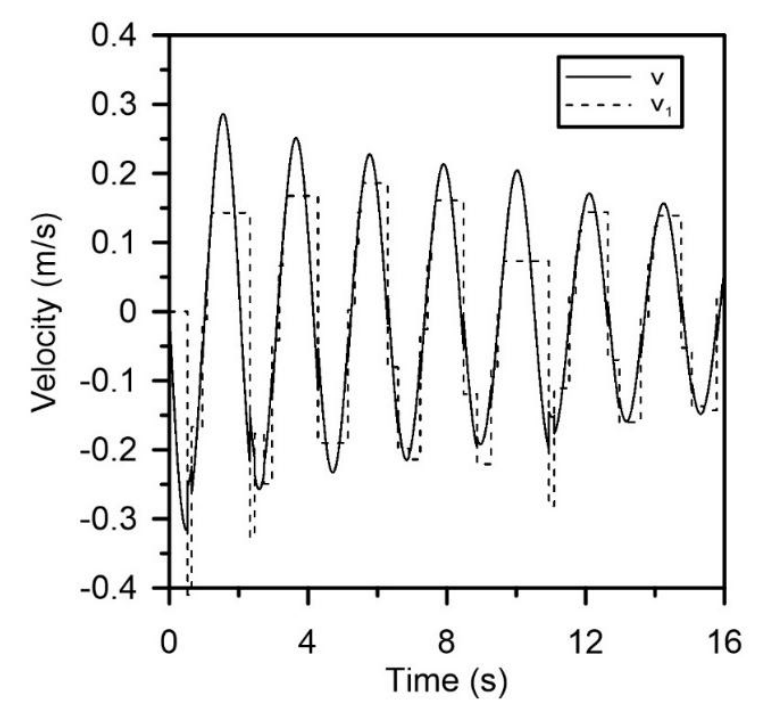

Fig. 4. Velocity in time domain ( $\mu=0.15, \gamma$ $=0.1$ and $\mathrm{e}=0.5$ )

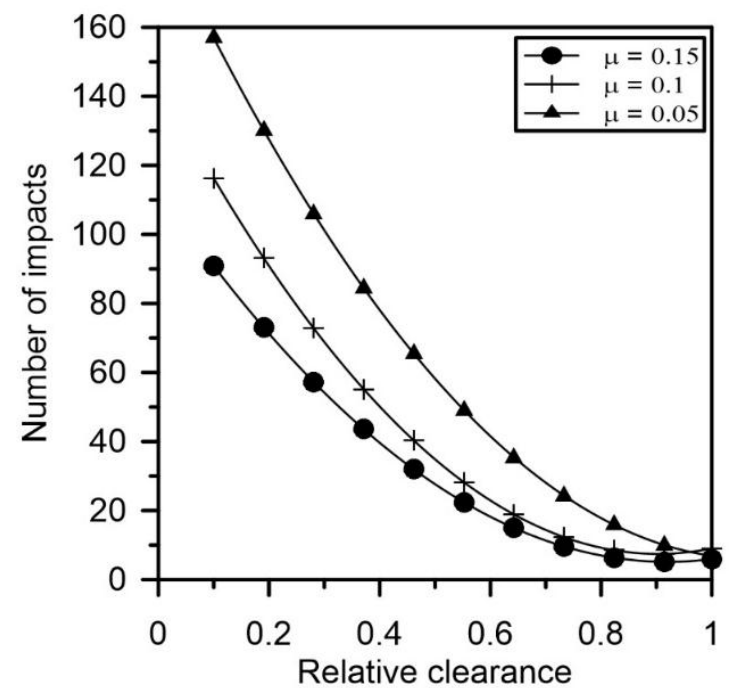

Fig. 6. Influence of relative clearance on the number of impacts for different mass ratios

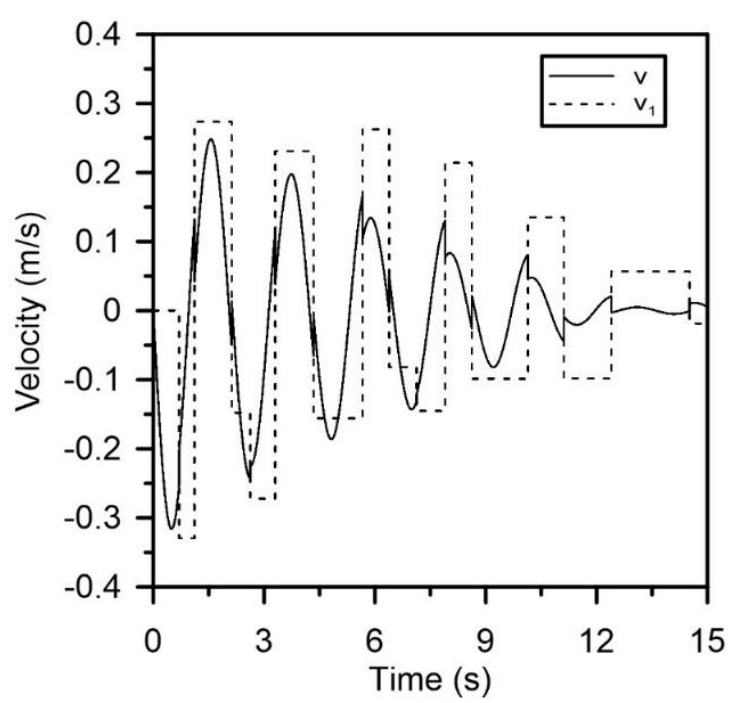

Fig. 5. Velocity in time domain ( $\mu=0.15, \gamma$ $=0.6$ and $\mathrm{e}=0.5$ )

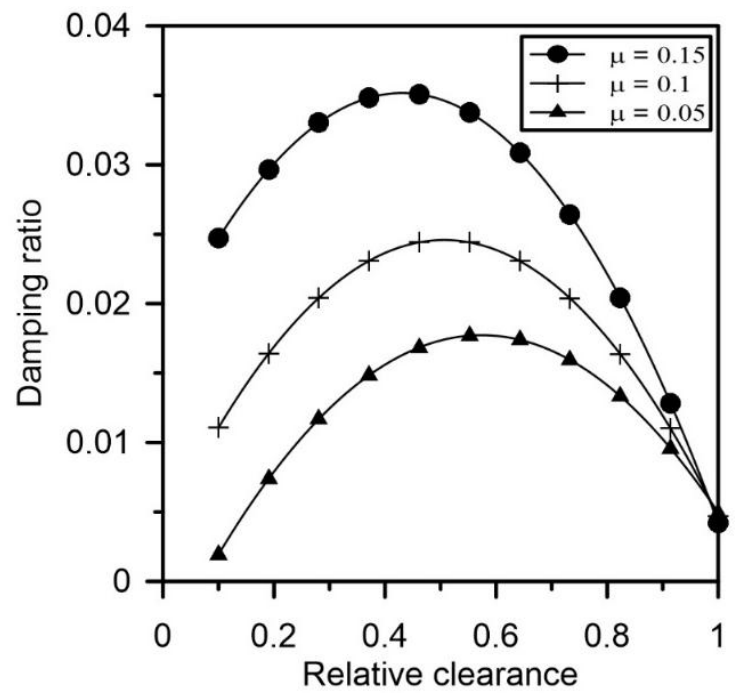

Fig. 7. Influence of relative clearance on the damping ratio for different mass ratios 


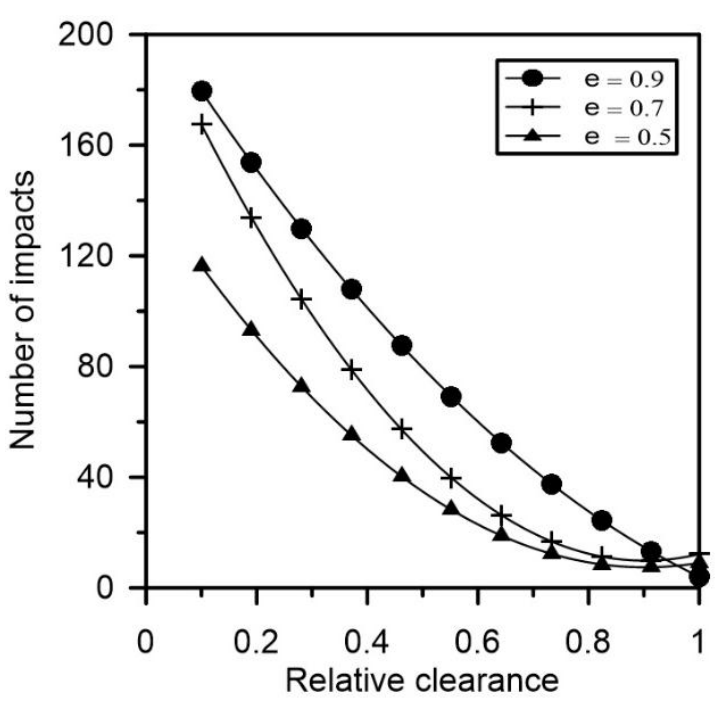

Fig. 8. Effect of relative clearance on the system damping for different restitution factors

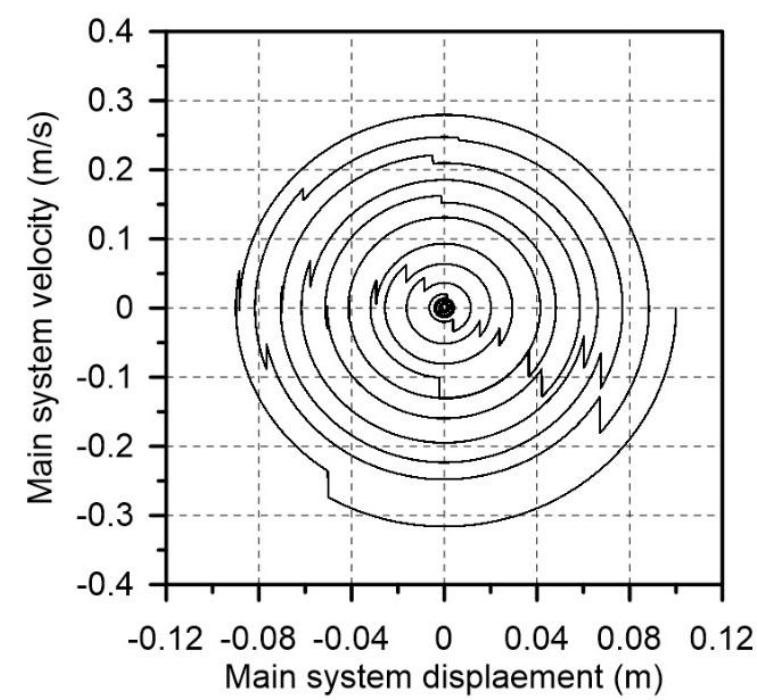

Fig. 10. Phase-plane plot of the main system $(\mu=0.1, \gamma=0.5$ and $\mathrm{e}=0.5)$

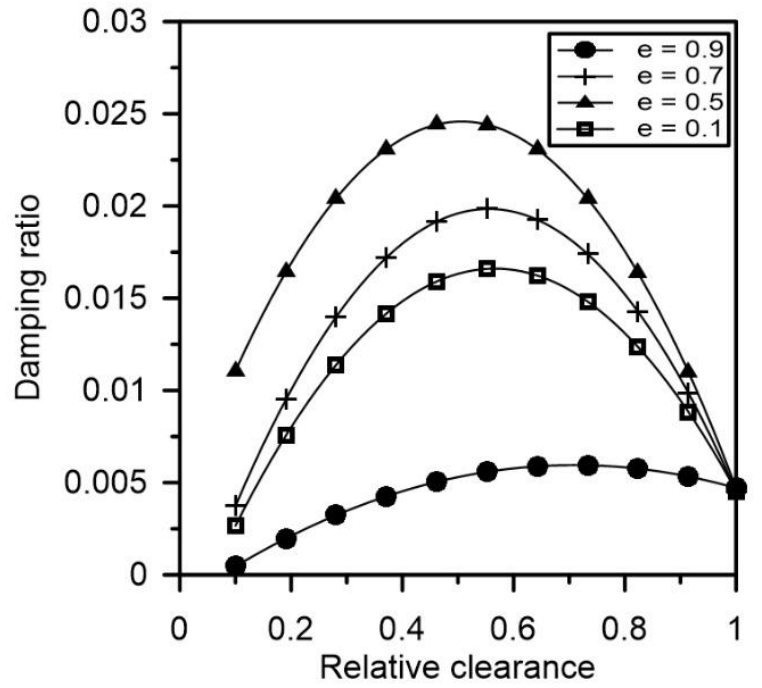

Fig. 9. Influence of relative clearance on the number of impacts for different restitution factors

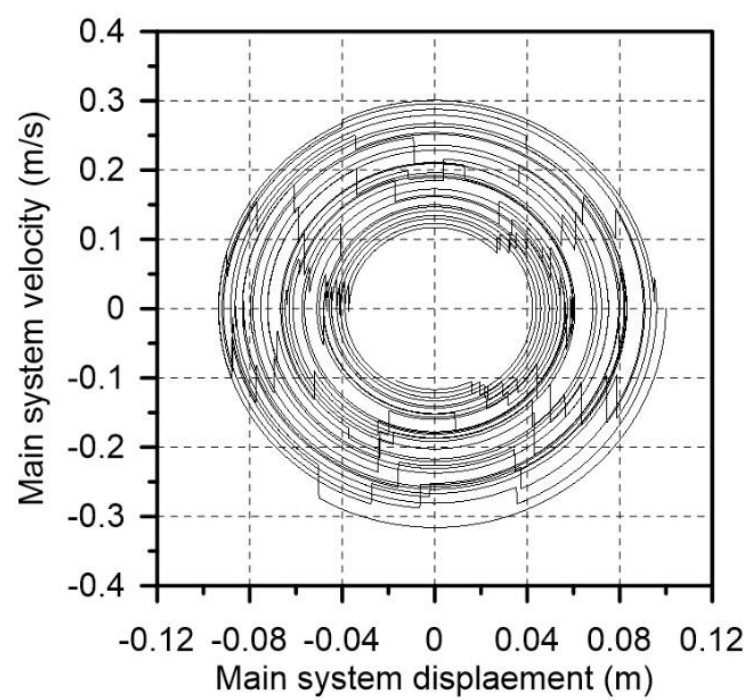

Fig. 11. Phase-plane plot of the main $\operatorname{system}(\mu=0.1, \gamma=0.5$ and $e=0.9)$ 


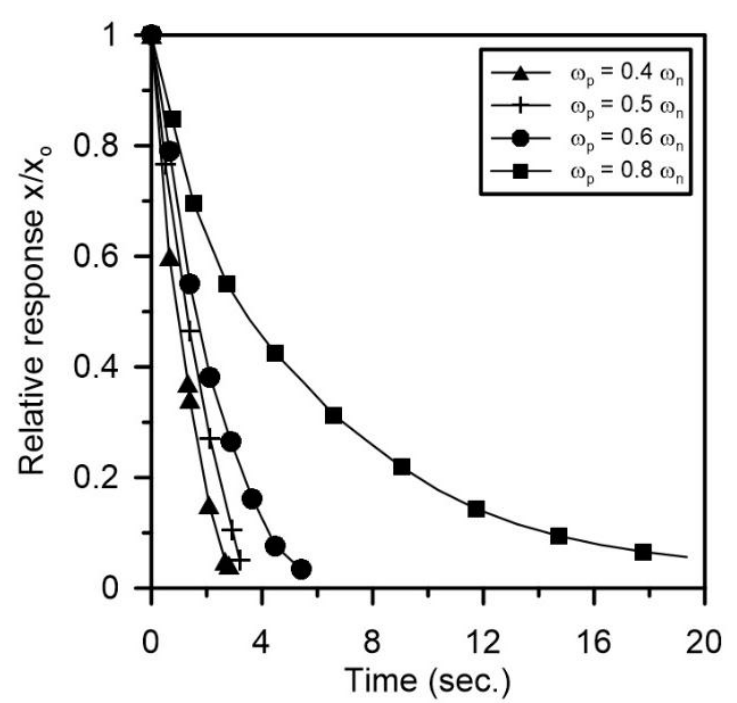

Fig.12. Effect of pendulum natural frequency on rate of decay

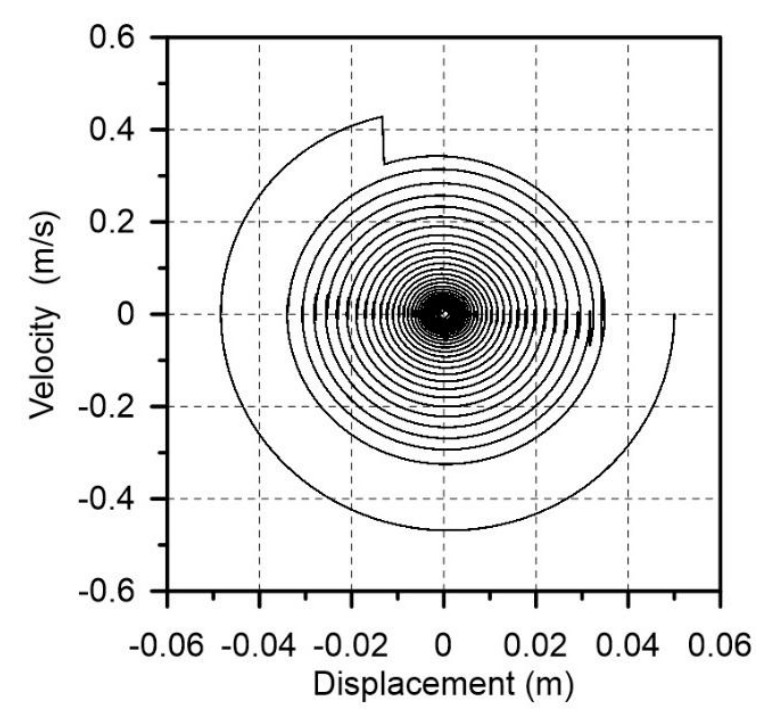

Fig.14. Phase-plane plot of the main $\operatorname{system}\left(\mu=0.1, \mathrm{e}=0.5\right.$ and $\left.\omega_{p}=0.8 \omega_{n}\right)$

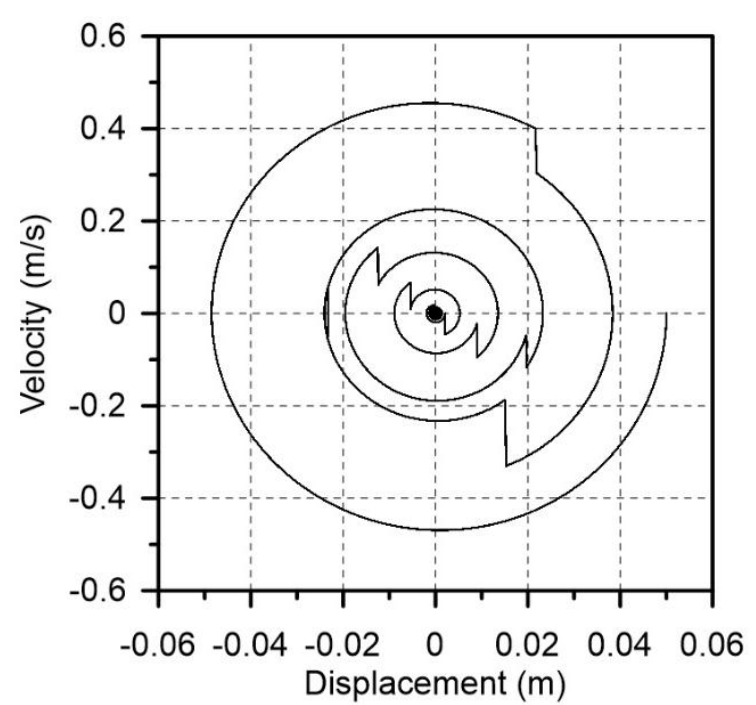

Fig.13. Phase-plane plot of the main $\operatorname{system}\left(\mu=0.1, \mathrm{e}=0.5\right.$ and $\left.\omega_{p}=0.4 \omega_{n}\right)$

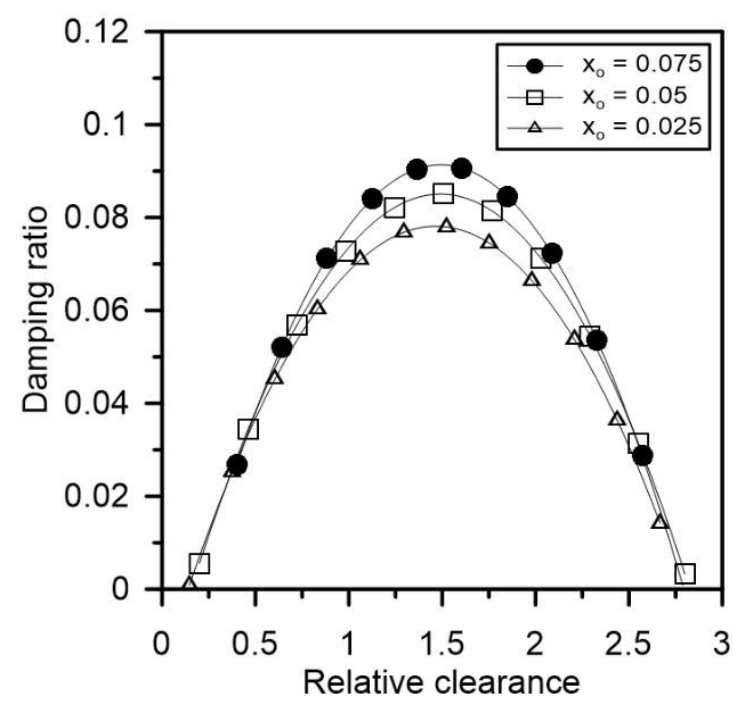

Fig. 15. Relative clearance effect on the damping ratio for different initial excitation amplitudes 


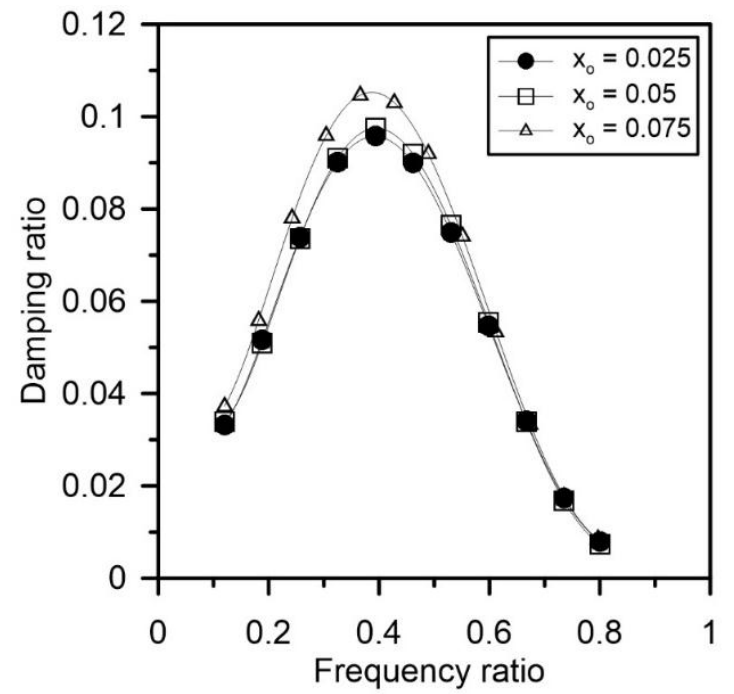

Fig. 16. Frequency ratio on the damping ratio for different initial excitation amplitudes

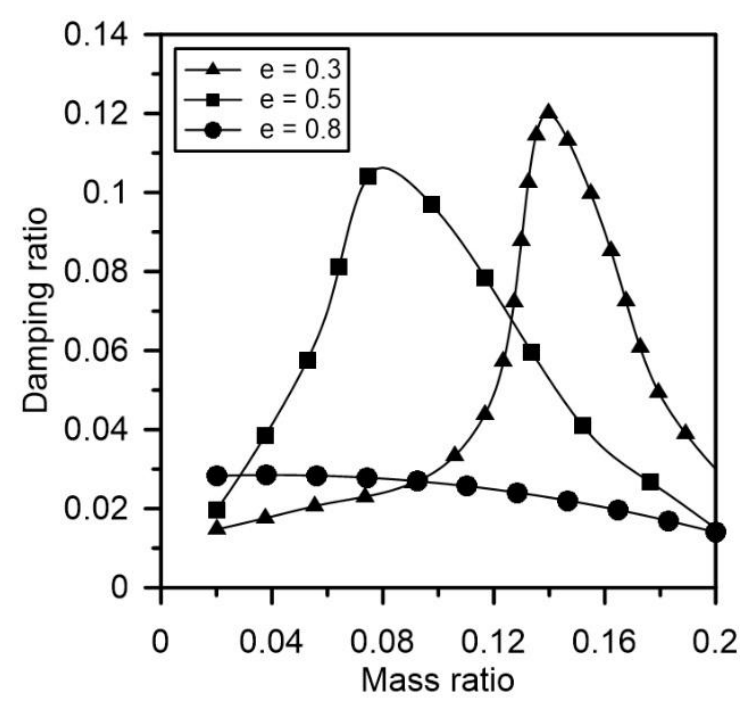

Fig. 17. Mass ratio effect on the damping ratio for different coefficient of restitutions

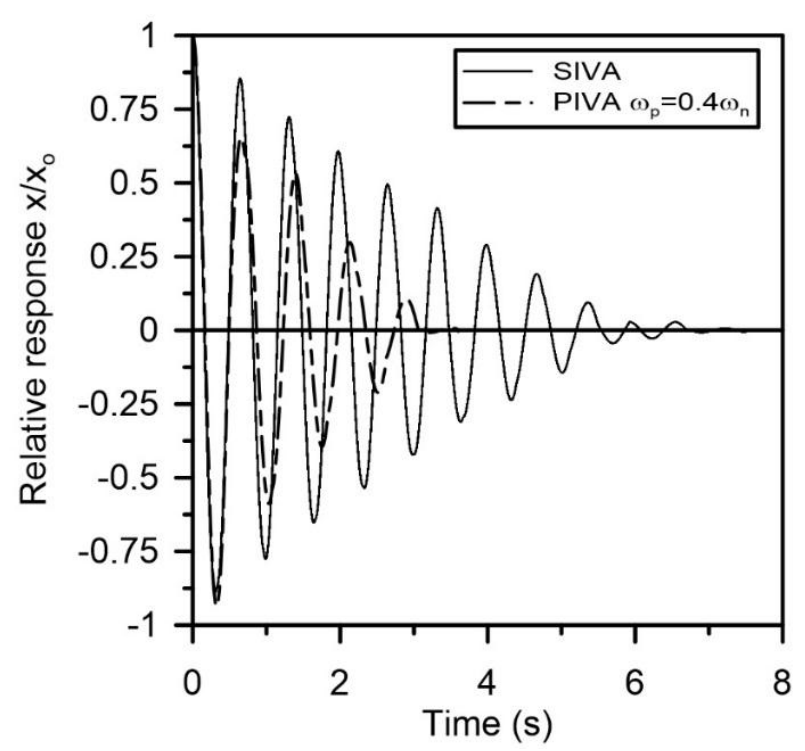

Fig. 18. Relative responses of main system in time domain for PIVA and SIVA 


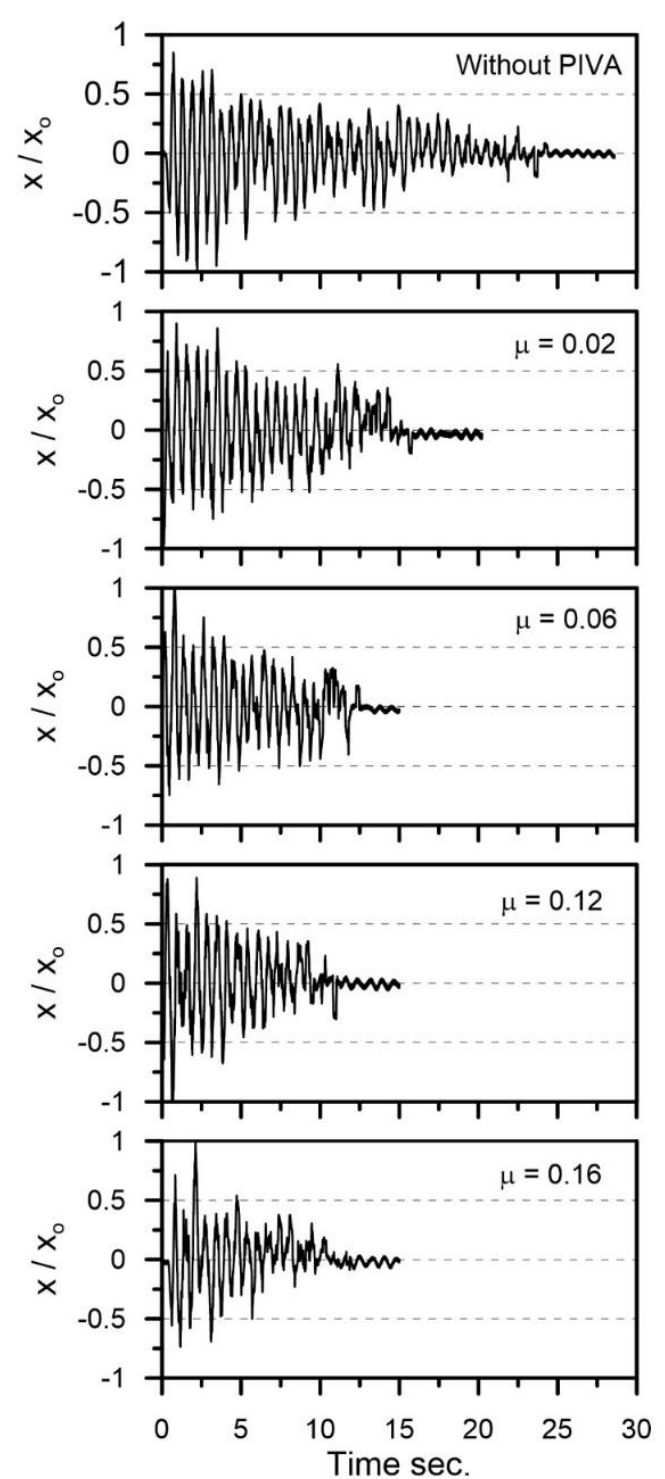

Fig. 19. Relative responses of the main system without and with PIVA for different mass ratios and $\gamma=1$.

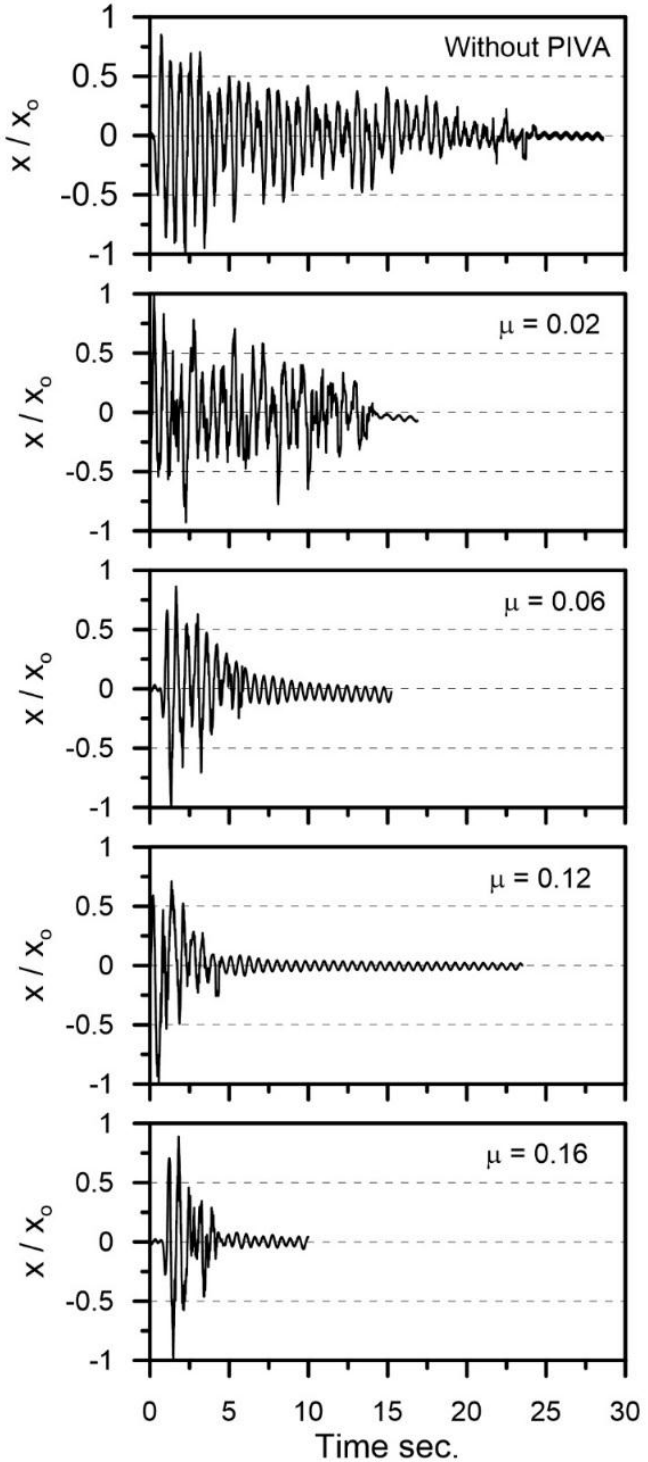

Fig. 20. Relative responses of the main system without and with PIVA for different mass ratios and $\gamma=1.4$. 


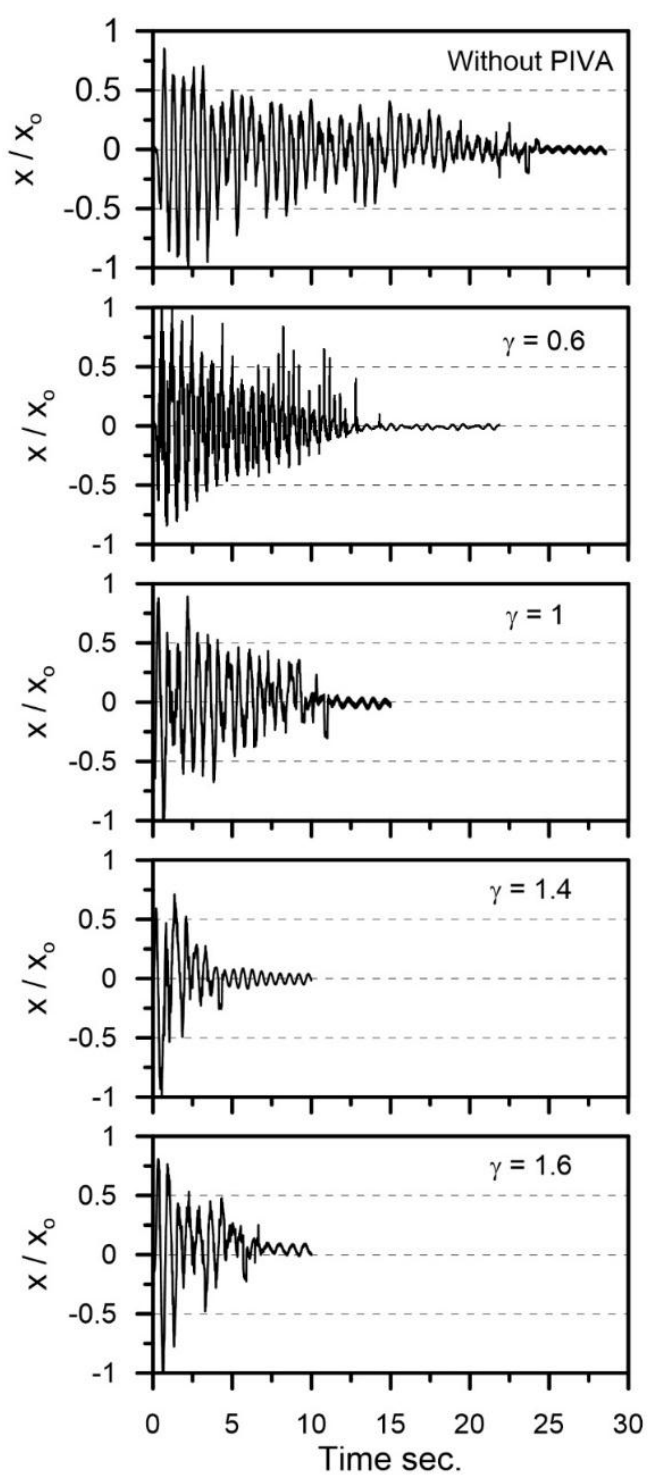

Fig. 21. Relative responses for different relative clearances and $\mu=0.12$.

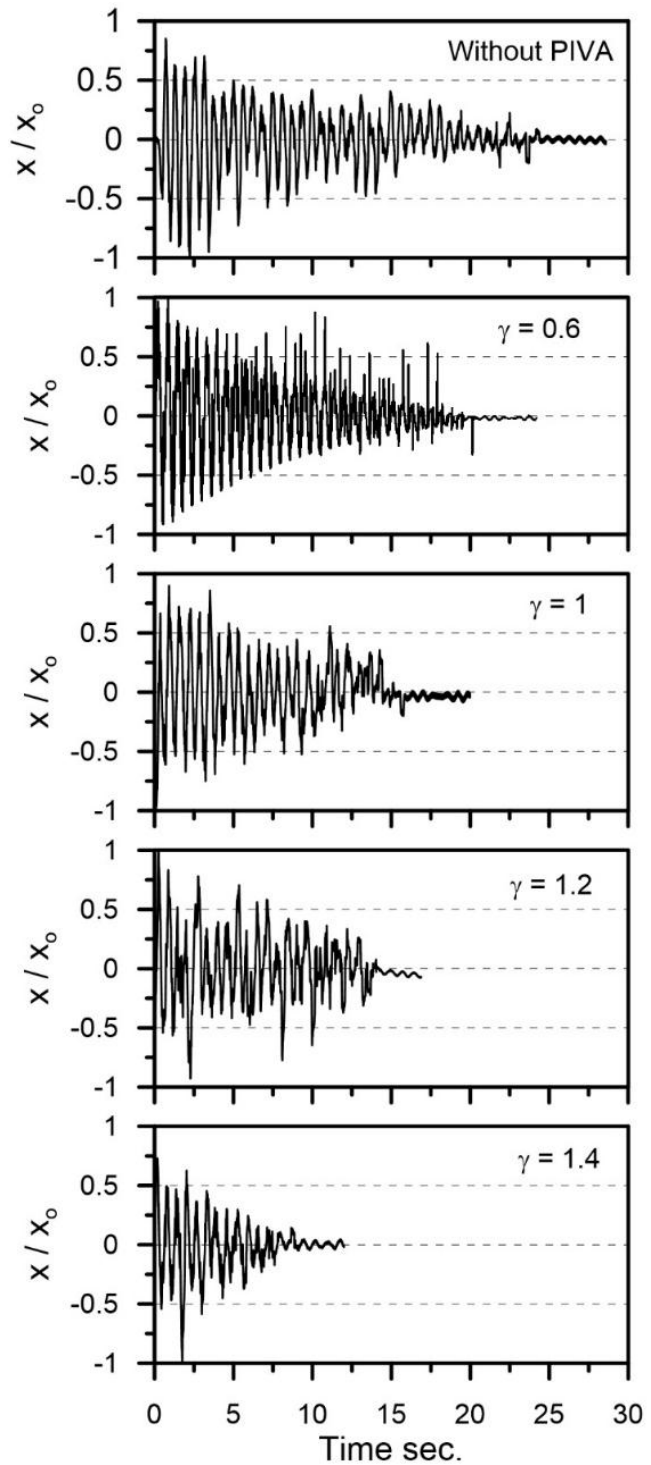

Fig. 22. Relative responses for different relative clearances and $\boldsymbol{\mu}=\mathbf{0 . 0 2}$ 